\title{
Influência dos diferentes estágios maturacionais na força de jovens praticantes de voleibol
}

The influence of diferente maturational stages in the strength of young volleyball practitioners

\author{
Rômulo Vasconcelos Teixeira* \\ Programa de Pós-Graduação em Educação Física, Centro de Ciências da Saúde, \\ Universidade Federal do Rio Grande do Norte (UFRN), Natal, RN, Brasil \\ romulovasconcelos11@hotmail.com
}

\author{
Matheus Peixoto Dantas \\ Departamento de Educação Física, Centro de Ciências da Saúde, \\ Universidade Federal do Rio Grande do Norte (UFRN), Natal, RN, Brasil \\ m.p.dantasef@gmail.com
}

Vanessa Carla Monteiro Pinto

Departamento de Educação Física, Centro de Ciências da Saúde, Universidade Federal do Rio Grande do Norte (UFRN), Natal, RN, Brasil

vanecmpinto@gmail.com

\author{
Breno Guilherme de Araújo Tinôco Cabra \\ Departamento de Educação Física, Centro de Ciências da Saúde, \\ Universidade Federal do Rio Grande do Norte (UFRN), Natal, RN, Brasil \\ brenotcabral@gmail.com
}

\section{Resumo:}

O presente estudo teve como objetivo analisar a influência dos diferentes estágios maturacionais nos componentes da aptidão física relacionados ao desempenho, especificamente a força explosiva de membros superiores e a altura do salto. Participaram do estudo 239 crianças, sendo 139 do sexo feminino e 100 do sexo masculino com idades entre 10 e 14 anos, praticantes de voleibol. Foram realizadas avaliações antropométricas e testes de capacidades físicas. $\mathrm{Na}$ análise estatística foi utilizada o teste de normalidade, Zscore da curtose e assimetria, houve aplicação do teste de Kruskall-Wallis, correlação de Spearman e análise de regressão linear. As meninas apresentaram resultados superiores na idade óssea quando comparadas aos meninos. As correlações entre a idade cronológica e a idade óssea demonstraram resultados similares. Concluiu-se que a idade óssea retrata uma correlação positiva com a força explosiva de membros superiores em ambos os sexos.

Palavras-chave: Puberdade, Jovens, Atletas, Aptidão física, Antropometria.

\section{Abstract:}

This study aimed to analyze the influence of different maturational stages on performance-related physical fitness components, specifically upper limb explosive strength and jump height. A total of 239 children participated in the study, all of which were volleyball practitioners: 139 were female and 100 were male, aged 10 to 14 years old. Anthropometric assessments and physical capacity tests were performed. For the statistical analysis, Z-score of kurtosis and asymmetry for normality test, and KruskallWallis test, Spearman's correlation and linear regression analysis were used. The girls presented superior results in bone age when compared to boys. Correlations between chronological age and bone age showed similar results. It was concluded that bone age shows a positive correlation with the explosive strength of upper limbs in both sexes.

KEYWORDs: Puberty, Young, Athletes, Physical fitness, Anthropometry. 


\section{INTRODUÇÃO}

O voleibol é uma modalidade esportiva que tem como característica o grande número de saltos realizados por partida (Sheppard, Gabbett \& Stanganelli, 2009). Além disso, foi demonstrado que a capacidade de salto vertical pode influenciar a capacidade de aspectos técnicos, como a performance de ataque, que é descrito como um dos mais importantes preditores de vitória em partidas competitivas (Challoumas \& Artemiou, 2018). Naturalmente, as características de alto nível são observadas em crianças e adolescentes no processo de seleção de talentos e, portanto, é necessário entender o comportamento motor de jovens praticantes de voleibol.

No treinamento com jovens atletas, a maturação e o crescimento são fatores extremamente importantes e relevantes a serem analisados, por serem processos dinâmicos correlacionados a uma extensa quantidade de mudanças em suas estruturas como um todo (Linhares et al., 2009). Diante disso, a ciência do esporte estuda eventos do desenvolvimento humano provenientes de três funções básicas dos seres vivos: crescimento, desenvolvimento e maturação (Seabra, Maia \& Garganta, 2001). Segundo Cabral et al., (2015) a maturação é um processo de desenvolvimento progressivo que incide no corpo do indivíduo de forma contínua e sistemática onde ocorre mudanças tanto físicas como biológicas.

Dessa forma, é notório que a maturação ocasiona mudanças morfológicas e metabólicas da infância até o completo estado maduro (Cole, Ahmed, Preece, Hindmarsh \& Dunger, 2015; Kaczor, Ziolkowski, Popinigis \& Tarnopolsky, 2005; Vandewalle et al., 2014). Além disso, são sugeridos que ao longo do processo de desenvolvimento, o percentual de fibras do tipo 2 e o tamanho do corte transversal da área do músculo aumentam (Lexell, Sjöström, Nordlund, \& Taylor, 1992), assim como a massa magra (Vandewalle et al., 2014) e hormônios com efeitos anabólicos (Cole et al., 2015). De fato, estudos tem mostrado uma relação entre o processo maturacional e o desenvolvimento de força (Cabral et al., 2016; Dantas, Barbosa, Silva, \& Cabral, 2016). Com isso, a identificação do momento exato em que ocorre os estágios maturacionais se torna extremamente relevante para o processo de treinamento. Dentre os métodos de avaliação da idade óssea, foi utilizado para diagnóstico da maturação biológica o proposto por (Cabral et al., 2016). Portanto, diante do avanço constante da ciência, torna-se necessário a utilização de métodos com maior validação externa, bem como os aspectos de ação entre eles, métodos esses que auxiliem na análise adiantada das particularidades e aptidões de cada indivíduo (Anfilo \& Shigunov, 2004).

Diante disso, o presente estudo teve como objetivo analisar a influência dos diferentes estágios maturacionais nos componentes da aptidão física relacionados ao desempenho, especificamente a força explosiva de membros superiores e altura do salto.

\section{Metodologia}

\section{Amostra}

Participaram do estudo 239 crianças, sendo 139 do sexo feminino e 100 do sexo masculino com idades entre 10 e 14 anos, praticantes de voleibol. Os sujeitos foram recrutados em um projeto esportivo durante o horário de treino cotidiano. Como critério de inclusão, foi adotado: a entrega do termo de consentimento livre e esclarecido (TCLE) e o termo de assentimento livre e esclarecido (TALE). E foram excluídos da amostra os sujeitos que apresentaram quaisquer limitações motoras e cognitivas que inviabilizassem a realização dos procedimentos da pesquisa.

O estudo foi aprovado pelo Comitê de Ética e Pesquisa da Universidade Federal do Rio Grande do Norte (CEP-UFRN), sob o parecer (protocolo no $1249937 / 2015$ ) em consonância com a declaração de Helsinque (2002) e resolução no 466/2012 do conselho nacional de saúde do Brasil. 


\section{Procedimentos}

Foram realizadas avaliações antropométricas e de capacidades físicas no mesmo dia. Inicialmente, as crianças foram direcionadas a uma sala própria para a realização das avaliações antropométricas. Dois pesquisadores com experiência prévia realizavam as avaliações (avaliador $1=$ Dobra cutânea e diâmetro ósseo; avaliador 2: massa, estatura e perímetro). Em seguida, os participantes eram direcionados para a quadra de treinamento, onde realizavam os testes físicos (arremesso de medicine ball e salto vertical contra movimento).

\section{Idade óssea}

Foi avaliada por meio do modelo preditor da idade óssea (Cabral et al., 2013b). A equação utiliza variáveis antropométricas para identificação da variação da idade óssea. [Idade óssea $=-11,620+7,004^{*}$ (estatura) $+1,226^{*}$ Dsexo $+0,749^{*}($ idade $\left.)-0,068^{*}(\operatorname{Tr})+0,214^{*}(\mathrm{Pcb})-0,588^{*}(\mathrm{Du})+0,388^{*}(\mathrm{Df})\right]$. Onde: para o sexo masculino: Dsexo $=0$, para o sexo feminino: Dsexo=1, Estatura $(\mathrm{m})$, Idade $($ anos $), \operatorname{Tr}=$ dobra cutânea triciptal $(\mathrm{mm}), \mathrm{Pcb}=$ perímetro corrigido de braço $(\mathrm{cm}), \mathrm{Du}=$ diâmetro de úmero $(\mathrm{cm}), \mathrm{Df}$ $=$ diâmetro de fêmur $(\mathrm{cm})$.

A estatura foi avaliada por meio de um estadiômetro com precisão de $0,1 \mathrm{~mm}$ (Sanny ES2020 ${ }^{\circ}$ ) com escala de 0,5 centímetros. A massa foi aferida através da balança antropométrica (filizola ${ }^{\circledR} 110$, com capacidade para $150 \mathrm{~kg}$ e divisões de $1 / 10$ de $\mathrm{kg}$ e precisão de 100 gramas). A dobra de tríceps foi avaliada com um adipômetro cientifico da marca Harpenden ${ }^{\circ}$ (Jon Bull Indicators Ltd.) com precisão de 0,2 mm. Para mensurar o perímetro corrigido do braço foi utilizado uma fita antropométrica metálica com precisão de $0,1 \mathrm{~mm}$ e os diâmetros ósseos do úmero e do fêmur foram mensurados com paquímetro antropométrico, ambos da marca Sanny. Todos os procedimentos foram realizados por dois avaliadores e seguiram rigorosamente as diretrizes da International Society for Advancement in Kinanthropometry - ISAK (Marfell-Jones, Stewart \& Olds, 2006).

\section{Maturação}

Para determinar a maturação, foi calculada a idade cronológica das crianças por meio da soma de todos os meses de vida divididos por 12. Após a determinação, foi realizada a subtração da idade óssea e da idade cronológica (IO-IC) e os valores foram estratificados em inferiores a -1 , entre $-1<\mathrm{e}\rangle+1$ e superiores a +1 e classificados como maturação acelerada, maturação adequada e maturação atrasada, respectivamente (Malina \& Bouchard, 2002).

\section{Instrumentos}

\section{Força explosiva de membros superiores}

Para a avaliação da FEMS, foi utilizado o teste de arremesso de medicine ball com a mesma pesando $2 \mathrm{~kg}$. O teste consiste em realizar um lançamento da bola com os membros superiores na maior distância possível. Para isso, os sujeitos sentavam-se com a coluna em total contato com a parede e pernas completamente estendidas no solo. A bola foi posicionada na altura do esterno e em uma única ação com os membros superiores, as crianças arremessavam a bola. O procedimento foi realizado duas vezes e a maior distância foi usada para análise (Safrit, 1995). 


\section{Altura do salto}

Para mensuração da altura do salto foi empregado o teste de salto vertical contra movimento com auxílio dos braços em uma plataforma de contato Cefise ${ }^{\bullet}$ conectada ao software Jump Test Pro 2.10. Foram realizados dois saltos com intervalo de dois minutos entre os mesmos e posteriormente foi computado o melhor resultado (Komi \& Bosco, 1978).

\section{Análise estatistica}

A normalidade da amostra foi testada por meio do teste de Kolmorov-Smirnov e pelo Z-score da curtose e assimetria. A comparação entre os estágios maturacionais foi realizada a partir do teste de Kruskall-Wallis. A relação das variáveis foi verificada a partir da correlação de Spearman e da análise de regressão linear. Foram adotados os limiares de (Cohen, 1988): $<0,1$ trivial; $0,1-0,3$ pequeno; 0,3 - 0,5 moderado; 0,5 0,7 forte; 0,7 - 0,9 muito forte; 0,9 - 0,99 quase perfeito; e 1,0 perfeito. Para todas as análises foi adotado o $\mathrm{p}<0,05$.

\section{Resultados E Discuss Ão}

A tabela 1 reporta diferença estatística entre meninos e meninas. Em relação a nossa amostra, identificamos que os meninos possuem idade óssea menor do que as meninas. Em adição, as meninas possuem estatura, massa e IMC superiores aos dos meninos. Em contrapartida, não houve diferença significativa para a FEMS e AS entre os grupos.

TABELA 1

Análise descritiva da amostra separada por sexo

\begin{tabular}{llll}
\hline & Meninos & Meninas & $\mathrm{P}$ \\
\hline IC (anos) & $12,10 \pm 1,90$ & $12,30 \pm 2,45$ & 0,181 \\
IO (anos) & $11,10 \pm 3,15$ & $12,50 \pm 4,63$ & 0,001 \\
Massa $(\mathrm{kg})$ & $38,30 \pm 17,60$ & $43,05 \pm 16,25$ & 0,011 \\
Estatura $(\mathrm{m})$ & $1,47 \pm 0,14$ & $1,52 \pm 0,15$ & 0,006 \\
$\mathrm{IMC}\left(\mathrm{kg} / \mathrm{m}^{2}\right)$ & $17,47 \pm 4,79$ & $18,89 \pm 5,02$ & 0,044 \\
FEMS (m) & $1,90 \pm 0,91$ & $2,05 \pm 1,19$ & 0,948 \\
$\mathrm{AS}(\mathrm{cm})$ & $25,10 \pm 8,5$ & $25 \pm 9,03$ & 0,256 \\
IC = Idade Cronológica. IO= Idade Ossea. IMC = Indice de Massa Corporal. FEMS \\
= Força Explosiva de Membros Superiores. AS = Altura do Salto.
\end{tabular}

As análises de correlação estão reportadas na tabela 2. Os dados do presente estudo reportam forte relação positiva entre a idade óssea e a FEMS em meninos $\left(r^{2}=36 \%\right)$ e meninas $\left(r^{2}=45 \%\right)$. Para a AS, identificamos relação positiva moderada em meninos $\left(r^{2}=19 \%\right)$ e meninas $\left(r^{2}=16 \%\right)$. 
TABELA 2

Correlação linear de Pearson entre a idade óssea e a FEMS e AS em ambos os sexos

\begin{tabular}{lcc}
\hline & Meninos & Meninas \\
\hline & $\mathrm{r}\left(\mathrm{r}^{2}\right)$ & $\mathrm{r}\left(\mathrm{r}^{2}\right)$ \\
\hline FEMS (m) & $0,602(0,36)$ & $0,703(0,45)$ \\
AS $(\mathrm{cm})$ & $0,437(0,19)$ & $0,456(0,16)$ \\
\hline
\end{tabular}

FEMS $=$ Força Explosiva de Membros Superiores. AS $=$ Altura do Salto .

Nossos achados demonstram que entre os meninos houve diferença dos sujeitos com maturação atrasada para os de maturação adequada e acelerada, mas não entre os sujeitos com maturação adequada para os de maturação acelerada. Em contrapartida, as meninas apresentaram diferenças entre as de maturação atrasada para as aceleradas e entre as adequadas para as aceleradas, mas não entre maturação atrasada e adequada (tabela 3).

TABELA 3

Teste de Kruskall-Wallis para comparação da altura do salto $(\mathrm{cm})$ entre os estágios maturacionais

\begin{tabular}{llll}
\hline & Atrasado & Adequado & Acelerado \\
\hline Meninos & $22,90 \pm 4,80^{* \#}$ & $27,20 \pm 8,15$ & $32 \pm 14,50$ \\
Meninas & $20,90 \pm 4,90^{\#}$ & $24 \pm 10^{\&}$ & $28 \pm 9$ \\
\hline${ }^{*} \mathrm{p}<0,05$ entre atrasado e adequado; ${ }^{*} \mathrm{p}<0,05$ entre atrasado e acelerado; ${ }^{-2} \mathrm{p}<0,05$ entre adequado e \\
acelerado.
\end{tabular}

A tabela 4 apresenta que entre os meninos houve diferença dos sujeitos com maturação atrasada para os de maturação adequada $(\mathrm{p}<0,001)$ e acelerada $(\mathrm{p}<0,007)$, por outro lado não ocorreu entre os sujeitos com maturação adequada para os de maturação acelerada. Em contrapartida, as meninas apresentaram diferenças entre as de maturação atrasada para as de maturação adequada $(\mathrm{p}<0,004)$ e acelerada $(\mathrm{p}<0,001)$ e entre as adequadas para as aceleradas $(\mathrm{p}<0,001)$.

TABELA 4

Teste de Kruskall-Wallis para comparação da FEMS (m) entre os estágios maturacionais

\begin{tabular}{llll}
\hline & Atrasado & Adequado & Acelerado \\
\hline Meninos & $1,68 \pm 0,45^{* \#}$ & $2,18 \pm 1,06$ & $2,93 \pm 2,44$ \\
Meninas & $1,25 \pm 0,64^{* \#}$ & $1,80 \pm 0,97^{\&}$ & $2,45 \pm 0,78$ \\
\hline
\end{tabular}

* diferença entre atrasado e adequado; \# diferença entre atrasado e acelerado; \& diferença entre adequado e acelerado.

A maturação biológica tem sido amplamente pesquisada por ser uma variável fundamental para o desenvolvimento de talentos. No presente estudo, identificamos uma relação similar entre a força explosiva e a idade óssea, onde a FEMS apresenta uma forte correlação e a AS apresenta uma moderada correlação em crianças de ambos os sexos. Tais resultados corroboram com outros estudos (Kalichman, Malkin, \& Kobyliansky, 2005; Malina, 2009), que relatam que o processo de maturação sofre ampla ação de diversos fatores tais como clima, nutrição, dentre outros, destacando-se o gênero ao longo do processo de crescimento e desenvolvimento ósseo. Nosso estudo indica que as meninas apresentam desenvolvimento ósseo superiores 
a os meninos, corroborando dessa forma com outras evidencias (Kishali, Imamoglu, Katkat, Atan, \& Akyol, 2006; Little \& Malina, 2007), as mesmas apontam que os aspectos maturacionais de meninas tendem a ser mais acelerados quando comparados a os meninos da mesma idade. Tais resultados podem ser atribuídos à ação hormonal, onde as meninas tendem a apresentar interferencia mais precocemente (Silva, Pelegrini, Petroski, \& Gaya, 2010). Também demonstramos que os sujeitos que possuem maturação atrasada desenvolvem menos força explosiva em relação aos seus pares com ritmo adequado e acelerado de maturação.

Evidências apontam que a maturação se relaciona com a aptidão física de jovens (Cabral, Cabral, Medeiros, Alcatara, \& Dantas, 2013a; Cabral et al., 2016; Dantas et al., 2016). De fato, modificações estruturais e metabólicas ocorrem ao longo do processo maturacional e sugerem aumento da produção de força muscular (Lexell et al., 1992; Kaczor et al., 2005; Cole et al., 2015). Os achados de Cabral et al., (2013a) apontam associação entre a idade óssea e os diversos aspectos físicos e motores tais como a força explosiva de membros superiores (FEMS) e a altura do salto (AS), tornando-se inegável a relevância dessa variável como sinalizadora de maturação. Em um estudo longitudinal com o objetivo de investigar a influência da idade cronológica e idade biológica no desenvolvimento de estudantes, foi demonstrado que o crescimento e a maturação biológica são aspectos relevantes a serem considerados (Machado \& Barbanti, 2007).

No nosso estudo, a idade óssea foi mais eficaz em predizer a FEMS em comparação com a AS. Em estudo mais recente, foram investigados 149 sujeitos, de ambos os sexos, sendo 55 meninos e 94 meninas, entre 8 e 14 anos, praticantes de voleibol, com o objetivo de analisar a correlação da idade óssea com as variáveis de aptidão física e antropométrica no esporte supracitado. Foi observado que a idade óssea apresentou uma positiva correlação com a força explosiva de membros superiores $(\mathrm{r}=0,585$; $\mathrm{p}<0,001)$ e altura do salto $(\mathrm{r}=$ 0,227; $\mathrm{p}<0,03)$ em jovens que praticam o esporte, dessa forma, tais resultados mostram ainda que sujeitos com maturação acelerada apresentam maiores níveis de força explosiva quando comparados com sujeitos da mesma idade com maturação atrasada (Cabral et al., 2016). Além disso, o presente estudo demonstrou uma produção de força linear entre os estágios maturacionais (Atrasado<Adequado<Acelerado). É comprovado que os indivíduos possuem ritmos de desenvolvimentos diferentes e que crianças de mesma idade cronológica podem estar em estágios diferentes de maturação (Malina, Rogol, Cumming, Silva, \& Figueiredo, 2015). Esses resultados sugerem que indivíduos de mesma idade que estejam com maturação acelerada, podem produzir mais força que seus pares com maturação atrasada. Nesse contexto, as particularidades inerentes a força e maturação possuem extrema relevância no que diz respeito aos diversos aspectos que influenciam na formação de atletas.

Nosso estudo possuiu algumas limitações que devem ser observadas no momento da análise dos resultados, como por exemplo, o caráter transversal do estudo que impede afirmações de causa e efeito.

\section{CONSIDERAÇÕES FINAIS}

Concluiu-se que a idade óssea, quando utilizada como parâmetro de maturação biológica, retrata uma forte correlação com a força explosiva de membros superiores e moderada correlação com a altura do salto em crianças de ambos os sexos. Além disso, sujeitos que apresentam desenvolvimento acelerado possuem maior nível de força quando comparados com seus pares atrasados. Dessa forma torna-se extremamente importante a análise das mais diversas variáveis no processo de seleção e orientação esportiva por parte dos treinadores, uma vez que, a maturação é uma das determinantes dos resultados esportivos.

\section{CONFLitos DE INTERESSE}

Os autores declaram não haver conflitos de interesse 


\section{Agradecimentos}

A Coordenação de Aperfeiçoamento de Pessoal de Nível Superior (Capes) pela concessão de bolsa de pós-graduação a Rômulo Vasconcelos Teixeira.

\section{REFERÊNCIAS}

Anfilo, M. A., \& Shigunov, V. (2004). Reflexões sobre o processo de seleção e preparação de equipes: O caso da seleção brasileira masculina de voleibol infanto-juvenil. Revista Brasileira de Cineantropometria e Desempenho Humano, 6(1), 17-25. Retirado de https://periodicos.ufsc.br/index.php/rbcdh/article/view/3843/16822.

Cabral, B. G. A. T., Aidar, F. J., Gama, D. M., Pinto, V. C. M., Rodrigues, L. B., \& Zacarkim, M. R. Dantas, P. M. S. (2015). Correlação de diferentes protocolos para verificação da idade óssea em crianças. Motricidade, 11(1), 4448. Retirado de https://www.researchgate.net/publication/275153920_Correlacao_de_Diferentes_Protocolo s_para_Verificacao_da_Idade_Ossea_em_Criancas.

Cabral, B. G., Cabral, S. A., Medeiros, R. M., Alcatara, T., \& Dantas, P. M. S. (2013a). Relação da maturação com a antropometria e aptidão física na iniciação desportiva. Relacao da maturacao com a antropometria e aptidao fisica na iniciacao desportiva., 9(4), 12-21. https://doi.org/10.6063/motricidade.9(4).689.

Cabral, B. G. A. T., Cabral, S. A. T., Vital, R., Lima, K. C., Alcantara, T., Reis, V. M., \& Dantas, P. M. S. (2013b). Equação preditora de idade óssea na iniciação esportiva através de variáveis antropométricas. Revista Brasileira de Medicina do Esporte, 19(2), 99-103. https://doi.org/10.1590/S1517-86922013000200005.

Cabral, S. A. T., Cabral, B. G. A. T., Pinto, V. C. M., Andrade, R. D., Borges, M. V. O., \& Dantas, P. M. S. (2016). Relação da idade óssea com antropometria e aptidão física em jovens praticantes de voleibol. Revista Brasileira de Ciencias do Esporte, 38(1), 69-75. https://doi.org/10.1016/j.rbce.2015.12.003.

Challoumas, D., \& Artemiou, A. (2018). Predictors of Attack Performance in High-level Male Volleyball Players. International Journal of Sports Physiology and Performance, 13(9), 1-23. https://doi.org/10.1123/ijspp.2018-0 125.

Cohen, J. (1988). Statistical power analysis for the behavioral sciences. Statistical Power Analysis for the Behavioral Sciences (2nd edn). https://doi.org/10.1234/12345678.

Cole, T. J., Ahmed, M. L., Preece, M. A., Hindmarsh, P., \& Dunger, D. B. (2015). The relationship between Insulinlike Growth Factor 1, sex steroids and timing of the pubertal growth spurt. Clinical Endocrinology, 82(6), 862869. https://doi.org/10.1111/cen.12682

Dantas, M. P., Barbosa, P. R. S., Silva, L. M., \& Cabral, B. G. A. T. (2016). Relação da idade óssea e cronológica com capacidades físicas na iniciação esportiva. Motricidade, 12(2), 28-34. Retirado de https://www.researchgate.net/profile/Matheus_Dantas2/publication/321034952_Relacao_da_idade_osse a_e_cronologica_com_capacidades_fisicas_na_iniciacao_esportiva/links/5a09aa62a6fdcc $8 \mathrm{~b} 547814 \mathrm{~d} 2 \mathrm{~d} / \mathrm{Relaca}$ o-da-idade-ossea-e-cronologica-com-capacidades-fisicas-na-iniciacao-esportiva.pdf.

Kaczor, J. J., Ziolkowski, W., Popinigis, J., \& Tarnopolsky, M. A. (2005). Anaerobic and aerobic enzyme activities in human skeletal muscle from children and adults. Pediatric Research, 57(3), 331-335. https://doi.org/10.1203 /01.PDR.0000150799.77094.DE.

Kalichman, L., Malkin, I., \& Kobyliansky, E. (2005). Association between physique characteristics and hand skeletal aging status. American Journal of Physical Anthropology, 128(4), 889-895. https://doi.org/10.1002/ajpa.20223.

Kishali, N. F., Imamoglu, O., Katkat, D., Atan, T., \& Akyol, P. (2006). Effects of menstrual cycle on sports performance. The International journal of neuroscience, 116(12), 1549-1563. https://doi.org/10.1080/002074 50600675217.

Komi, P. V., \& Bosco, C. (1978). Utilization of stored elastic energy in leg extensor muscles by men and women. Medicine and science in sports, 10(4), 261-265. Retirado de https://www.researchgate.net/publication/223911 62_Utilization_of_stored_elastic_energy_in_leg_extensor_muscles_by_men_and_women. 
Lexell, J., Sjöström, M., Nordlund, a S., \& Taylor, C. C. (1992). Growth and development of human muscle: a quantitative morphological study of whole vastus lateralis from childhood to adult age. Muscle \& nerve, 15(3), 404-409. https://doi.org/10.1002/mus.880150323.

Linhares, R. V., Matta, M. de O., Lima, J. R. P., Dantas, P. M. S., Costa, M. B., \& Fernandes Filho, J. (2009). Efeitos da maturação sexual na composição corporal, nos dermatóglifos, no somatótipo e nas qualidades físicas básicas de adolescentes. Arquivos brasileiros de endocrinologia e metabologia, 53(1), 47-54. https://doi.org/10.1590/ S0004-27302009000100008 [pii].

Little, B. B., \& Malina, R. M. (2007). Gene-environment interaction in skeletal maturity and body dimensions of urban Oaxaca Mestizo schoolchildren. Annals of Human Biology, 34(2), 216-225. https://doi.org/10.1080/0 3014460601144011.

Machado, D. R. L., \& Barbanti, V. J. (2007). Maturação esquelética e crescimento em crianças e adolescentes. Revista Brasileira de Cineantropometria e Desempenho Humano, 9(1), 12-20. Retirado de https://periodicos.ufsc.br/in dex.php/rbcdh/article/viewFile/4025/3411.

Malina, R., \& Bouchard, C. (2002). Atividadefísica do atleta jovem: do crescimento à maturação.(Vol 9). São Paulo. Roca

Malina, R. M. (2009). Ethnicity and biological maturation in sports medicine research. Scandinavian journal of medicine \& science in sports, 19(1), 1-2. https://doi.org/10.1111/j.1600-0838.2009.00910.x.

Malina, R. M., Rogol, A. D., Cumming, S. P., Silva, M. J. C., \& Figueiredo, A. J. (2015). Biological maturation of youth athletes: Assessment and implications. British Journal of Sports Medicine, 49(13), 1-10. https://doi.org/10.11 36/bjsports-2015-094623.

Marfell-Jones, M. Stewart, A. Olds, T. (2006). Kinanthropometry IX: Proceedings of the 9th International Conference of the International Society for the Advancement of Kinanthropometry. International Society for the Advancement of Kinanthropometry. Potchefstroom, South Africa: Routledge.

Safrit, M. J. (1995). Complete guide to youth fitness testing. Champaign: Human Kinetics.

Seabra, A. Maia, J. A. Garganta, R. (2001). Crescimento, maturação, aptidão física, força explosiva e habilidades motoras específicas. Estudo em jovens futebolistas e não futebolistas do sexo masculino dos 12 aos 16 anos de idade. Revista Portuguesa de Ciências do Desporto, 1(2), 22-35. https://doi.org/10.5628/rpcd.01.02.22.

Sheppard, J. M. Gabbett, T. J. Stanganelli, L. C. R. (2009). An Analysis of Playing Positions in Elite Men's Volleyball: Considerations for Competition Demands and Physiologic Characteristics. Journal of Strength and Conditioning Research, 23(6), 1858-1866. https://doi.org/10.1519/JSC.0b013e3181b45c6a.

Silva, D. A. S., Pelegrini, A., Petroski, E. L., \& Gaya, A. C. A. (2010). Comparison between the growth of Brazilian children and adolescents and the reference growth charts: data from a Brazilian project. Jornal de Pediatria, 86(2), 115-120. https://doi.org/10.2223/JPED.1975.

Vandewalle, S., Taes, Y., Fiers, T., Toye, K., Van Caenegem, E., Roggen, I., ... Kaufman, J. M. (2014). Associations of sex steroids with bone maturation, bone mineral density, bone geometry, and body composition: a cross-sectional study in healthy male adolescents. The Journal of clinical endocrinology and metabolism, 99(7), 1272-1282. htt ps://doi.org/10.1210/jc.2013-3887. 